\title{
ANÁLISE DO EFEITO DA GINÁSTICA LABORAL NA QUALIDADE DE VIDA DE FUNCIONÁRIOS DE EMPRESAS DE CALL CENTER
}

DOI: 10.48140/digitaleditora.2020.002.16

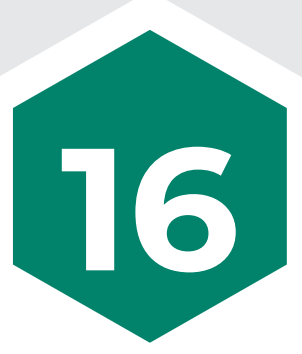

RESUMO

Objetivos: Desta maneira, o presente estudo tem como objetivo investigar por meio de uma pesquisa bibliográfica a análise do efeito da ginástica laboral na qualidade de vida de funcionários de empresas de call center.

Métodos: Trata-se de um estudo realizado por meio de pesquisa bibliográfica, constituído de artigos científicos. A coleta de dados sobre o efeito da ginástica laboral na qualidade de vida de funcionários de empresas de call center que será realizado a partir de artigos científicos por meio da ferramenta de pesquisa Google acadêmico. O estudo foi executado buscando apresentar a importância sobre o assunto, através de alguns autores, por meio de artigos dos últimos 18 anos.

Resultados: Os resultados de todos os estudos foram significamente positivos. Os trabalhadores tiveram um aumento do rendimento nas atividades diárias no trabalho, como também diminuição do estresse físico e mental, vícios posturais e lesões.

Conclusão: A Ginástica Laboral influencia na melhora das condições do bem-estar global dos indivíduos trabalhadores de empresas de teleatendimento.
Klenio Miguel de Carvalho

Graduando em Educação Física pela AESPI - Ensino Superior do Piauí

Teresina - Piauí

https://orcid.org/0000 0001-8802-1567

\section{Maria Victória Sants Silva}

Graduanda em Educação Física pela AESPI - Ensino Superior do Piauí

Teresina - Piauí

iD

\section{Hisabel Pereira de Araújo}

Educadora Física, Especialista e Professora da Faculdade AESPI Ensino Superior do Piauí Teresina - Piauí 


\section{ANALYSIS OF THE EFFECT OF LABOR \\ GYMNASTICS ON THE QUALITY OF \\ LIFE OF EMPLOYEES OF CALL CENTER \\ COMPANIES \\ DOI: 10.48140/digitaleditora.2020.002.16}

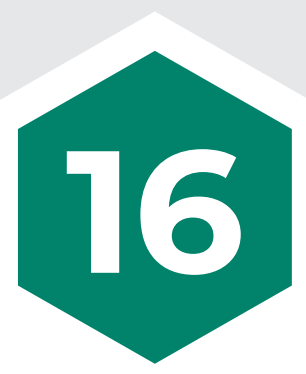

\section{ABSTRACT}

Recebido em: 10/12/2020

Aprovado em: 28/12/2020

Conflito de Interesse: não

Suporte Financeiro: não houve
Objectives: In this way, the present study aims to investigate through of a bibliographic research to analyze the effect of labor gymnastics on the quality of life of employees of call center companies.

Methods: This is a study conducted through bibliographic research, consisting of scientific articles. The collection of data on the effect of occupational gymnastics on the quality of life of employees of call center companies that will be carried out from scientific articles through the Google academic research tool. The study was carried out seeking to present the importance on the subject, through some authors, through articles from the last 18 years.

Results: The results of all studies were significantly positive. Workers had an increase in income in daily activities at work, as well as a decrease in physical and mental stress, postural addictions and injuries.

Conclusion: Labor Gymnastics influences the improvement of the conditions of global well-being of individuals working in call center companies. 


\section{(8) \\ INTRODUÇÃO}

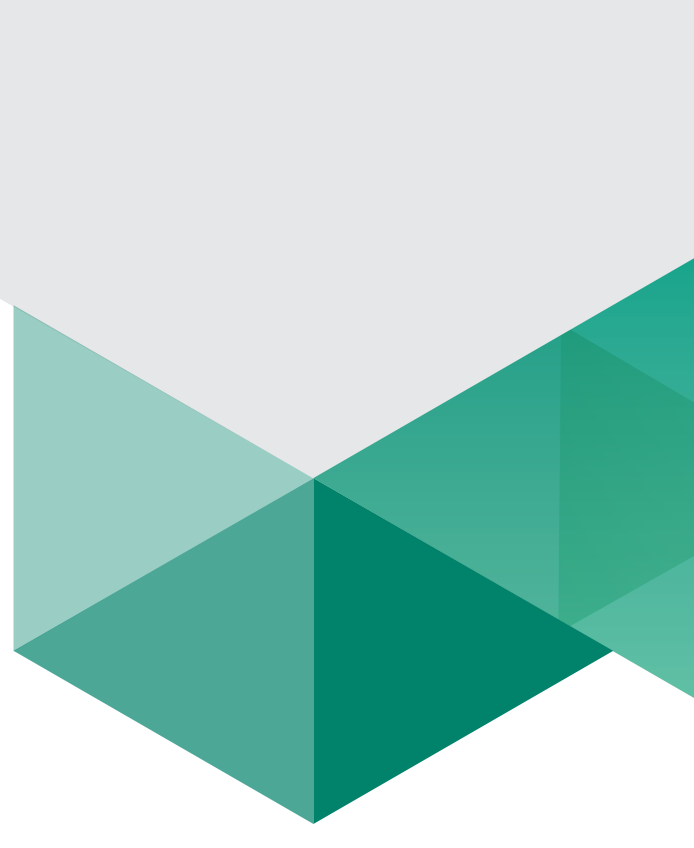

Considerando que, segundo o Instituto Brasileiro de Geografia e Estatística (IBGE) (2010), o agente de telemarketing é o funcionário que mais se estressa em função de seu ofício no Brasil, o que mais é autorizado pelo INSS a sair de licença saúde, o que mais se aposenta com rapidez, e o que mais desenvolve transtornos psíquicos, deve-se considerar também que as pausas destinadas à atividade física, são descansos não só para os movimentos repetitivos que o funcionário executa, mas também, para a mente, de seus protocolos e atendimentos estressantes. As empresas preocupadas com o bem-estar dos funcionários têm adotado estratégias tais como a realização de atividades de ginástica laboral (MENDES; LEITE, 2004), como forma de prevenção e promoção de saúde do trabalhador, devido ao seu baixo custo de aplicação e possíveis benefícios na saúde dos funcionários (TSCHOEKE; COSTA, 2015).

Como descrito por Silva (2004), vários estudos conseguem demonstrar e apontar sintomas de ordem física e mental em trabalhadores na atividade de telefonia, sendo que os aspectos psicoativos e relacionais recebem um enfoque diferenciado. Os principais efeitos negativos do trabalho dos tele atendentes são: fadiga visual, distúrbios do sono, sintomas digestivos gerais, distúrbios da personalidade e da vida relacional interferindo, inclusive, na vida fora do trabalho.

Ações que estimulem a prática da atividade física no ambiente de trabalho são bem-vindas, na perspectiva de promover a qualidade de vida do trabalhador e reduzir as despesas do empreendedor em função do afastamento temporário ou permanente de seus funcionários. As exigências atuais quanto à produtividade em empresas tornam tais ambientes de trabalho cada vez mais estressantes, com impactos negativos na motivação dos trabalhadores e no desempenho de suas funções. (ROSSATO et al., 2011).

Todo programa de ginástica laboral deve propiciar principalmente a mudança individual, que acarretará benefícios pessoais e coletivos, a partir de melhorias no ambiente ocupacional. Além disso, a ginástica laboral proporciona maior disposição para outras atividades do cotidiano, como atividades em família, com amigos e até mesmo a prática de atividade física fora do expediente de trabalho (ROSSATO et al., 2011).

Existem várias denominações para a Ginástica Laboral, como: Ginástica na empresa, ginástica matinal, ginástica corretiva e ginástica no trabalho. São conhecidas dessa forma por estarem relacio- 
nadas ao sistema musculoesquelético, prevenindo possíveis distúrbios osteomusculares relacionados ao trabalho (DORT), também conhecidos por doenças ocupacionais. Podem ser classificadas como: Ginástica Laboral Compensatória, realizada antes do expediente de trabalho, Ginástica de Pausa às realizadas durante o trabalho e Ginástica de Relaxamento às praticadas após as atividades laborais (ZILLI, 2002 p.57).

É preciso investigar o quanto a Ginástica Laboral (G.L.) pode ser uma ferramenta eficaz para promoção da saúde e conscientização à prática de atividade física, pois a sua prática busca um melhor equilíbrio biopsicossocial podendo influenciar de maneira positiva na prevenção de distúrbios osteomusculares como as LER/DORT que vem acarretando prejuízos à saúde de muitos trabalhadores de várias categorias profissionais em todo mundo (OLIVEIRA \& SAMPAIO, 2008).

Associando-se a Ginástica Laboral à saúde em um contexto onde o ambiente de trabalho e o estilo de vida estão interligados, observa-se a necessidade de um programa de atividade física, onde o investimento tem sido um retorno satisfatório para ambas as partes (empresa e empregados), buscando mudanças mais humanizadas no processo de trabalho e na saúde do trabalhador, abrindo espaço para programas de qualidade de vida e prevenção de doenças no ambiente de trabalho e até mesmo o incentivo às práticas de exercícios físicos fora da jornada do mesmo, proporcionando assim mudanças em seus hábitos cotidianos (CARVALHO, 2003).

Para Cañete (1996, p.16) a GL "quando implantada por convicção da cúpula da empresa e valendo-se de profissionais competentes mostra que é capaz não só de proporcionar aumento de produtividade, mas principalmente ela contribui para um acréscimo significativo na qualidade do trabalho".

Neste sentido, este trabalho apresentou a seguinte problemática: O que a ginástica proporciona aos seus usuários? Para isso, essa pesquisa teve como objetivos específicos, analisar os efeitos da ginástica laboral em funcionários de diferentes empresas de Call Center. E como objetivo geral, analisar os benefícios adquiridos através da implementação da ginástica laboral nas empresas de call center. Este estudo teve como propósito realizar uma revisão de literatura sobre o desempenho da ginástica laboral na melhora da qualidade de vida de trabalhadores de Call Center.

O presente estudo justifica-se pelo conhecimento de que a ginástica laboral tem a capacidade de proporcionar o aumento da qualidade de vida de trabalhadores de diferentes empresas de tele atendimento. Com isso, os profissionais atuantes na área da Saúde do Trabalhador, como os profissionais de educação física e fisioterapeutas, utilizam a ginástica laboral como forma de precaução e tratamento desses distúrbios.

\section{METODOLOGIA IIPO DE ESTUDO}

O presente estudo trata-se de uma revisão de literatura. Os critérios de elegibilidade foram artigos que tivessem como tema principal a ginástica laboral como forma de aquisição de condições para o bem-estar global dos indivíduos em empresas de Call center. A pesquisa analisou a ginástica laboral como intervenção da qualidade de vida pré e pós interferência. A pesquisa foi realizada através da ferramenta de pesquisa Google Acadêmico. Foram selecionados textos completos, em língua portuguesa, com os termos: Empresas de Call center, Ginástica Laboral e Qualidade de Vida. 


\section{METODOLOGIA | CRITÉRIOS DE INCLUSÃO E EX- CLUSÃO}

Estudos que analisaram a efeito da ginástica laboral na qualidade de vida de funcionários de empresas de call center. Artigos em português, com texto completo disponível na plataforma de dados nos últimos 18 anos. Exclusão, os estudos que não analisaram estes aspectos.

\section{PROCEDIMENTOS PARA COLETAS DE DADOS}

Para a realização desta pesquisa foi feito um levantamento bibliográfico para seleção dos estudos na base de dados online Google Acadêmico, onde foram selecionados artigos por meio das seguintes associações:

1ㅇ associação: Ginástica laboral na qualidade de vida.

2o associação: Funcionários de empresas de call center.

\section{ANÁLISE DOS DADOS}

Após o apanhado de dados, foi realizada uma análise de todos os estudos a fim de obter evidencias e resultados que mais fundamentaram o assunto aprofundado. Foi executada a leitura de todo o material designado e reunido as principais bases de dados, que buscaram estabelecer uma compreensão e ampliar o conhecimento sobre o tema estudado para que seja elaborado o referencial teórico.

\section{RESULTADOS E DISCUSSÃO}

Nesta revisão narrativa foram incluídos 4 artigos, foram excluídos 07 artigos por não atenderem os critérios propostos: artigos completos referentes à pesquisa (O efeito da ginastica laboral na qualidade de vida de funcionários de empresas de Call center), idioma (português), tempo do artigo (artigos publicados nos últimos 18 anos). 
Distribuição dos autores, base de dados, objetivos e resultados.

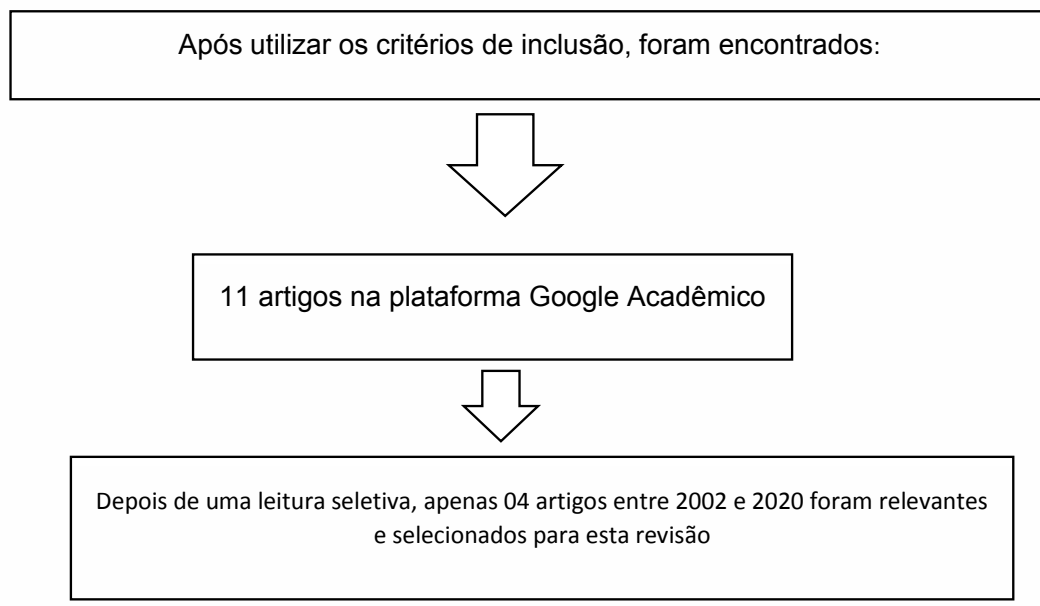

QUADRO 1. Distribuição dos autores, base de dados, objetivos e resultados.

\begin{tabular}{|c|c|c|}
\hline ANO & DADOS & OBJETIVO \\
\hline $\begin{array}{l}\text { Resen- } \\
\text { de ET } \\
\text { ALL,2006 }\end{array}$ & $\begin{array}{l}\text { Google } \\
\text { Acadêmico }\end{array}$ & $\begin{array}{l}\text { O presente trabalho teve como ob- } \\
\text { jetivos verificar a influência de um } \\
\text { programa de GL sobre as queixas dos } \\
\text { funcionários de um teleatendimen- } \\
\text { to e comparar os resultados da GL } \\
\text { quando aplicada por monitores trei- } \\
\text { nados (funcionário) e quando aplica- } \\
\text { da por fisioterapeutas. }\end{array}$ \\
\hline $\begin{array}{l}\text { Benevi- } \\
\text { des, } 2017\end{array}$ & $\begin{array}{l}\text { Google } \\
\text { Acadêmico }\end{array}$ & $\begin{array}{l}\text { Verificar na literatura, qual a efeti- } \\
\text { vidade dos programas de ginástica } \\
\text { laboral realizados em empresas de } \\
\text { teleatendimento sobre a qualidade } \\
\text { de vida dos funcionários e quais os } \\
\text { possíveis benefícios físicos em rela- } \\
\text { ção à postura e dores musculares. }\end{array}$ \\
\hline $\begin{array}{l}\text { Isaías, } \\
2015\end{array}$ & $\begin{array}{l}\text { Google } \\
\text { Acadêmico }\end{array}$ & $\begin{array}{l}\text { Investigar o que a literatura descreve } \\
\text { sobre a implicação da prática da Gi- } \\
\text { nástica Laboral na percepção de dor } \\
\text { de funcionários de teleatendimento. }\end{array}$ \\
\hline
\end{tabular}

Antes da GL 50\% dos funcionários do turno da manhã estavam satisfeitos com sua saúde e ao final do programa este número subiu para $60 \%$. Ao passo que no turno da tarde, eram $29 \%$ dos funcionários satisfeitos com a sua saúde no início e $57 \%$ ao final dos 04 meses (Figura 1). Com relação à satisfação com o trabalho antes da GL, 40\% dos funcionários da manhã e 43\% dos da tarde relataram estar satisfeitos, e ao final do programa estes valores subiram para 50\% cada. É necessário salientar que o programa de GL a ser implantado nas empresas de teleatendimento, deve respeitas as individualidades e as necessidades dos funcionários que desempenham esta função, e é indispensável que sejam realizados por profissionais da área de Educação Física com conhecimentos técnicos suficientes e que compreendem que essas ações são voltadas para a saúde e principalmente para a qualidade de vida, sobretudo pela qualidade de vida no trabalho dos praticantes.

A dor é uma das principais responsáveis por afastar o profissional do exercício de sua função. Foi verificado que o sexo feminino é mais suscetivel a doenças ocupacionais do que o sexo masculino e que a faixa etária dos trabalhadores das centrais de teleatendimento ficam entre 20 e 30 anos. A constatação foi feita após a análise dos artigos selecionados para esta pesquisa.

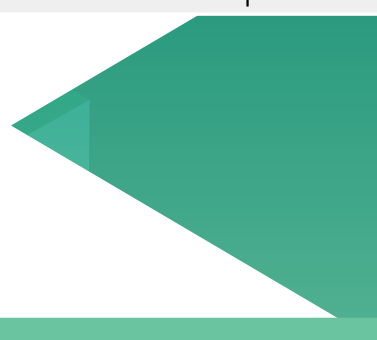




\begin{tabular}{|c|c|c|c|}
\hline $\begin{array}{l}\text { Souza, } \\
2012\end{array}$ & $\begin{array}{l}\text { Google } \\
\text { Acadêmico }\end{array}$ & $\begin{array}{l}\text { Discutir as implicações da saúde e os } \\
\text { fatores de risco relacionados ao am- } \\
\text { biente laboral do teleoperador e tem } \\
\text { como objetivo analisar através de } \\
\text { uma revisão bibliográfica os fatores } \\
\text { de risco relacionados aos profissio- } \\
\text { nais de call center. }\end{array}$ & $\begin{array}{l}\text { A análise bibliográfica identificou diversos } \\
\text { fatores de risco relacionados com o traba- } \\
\text { lho em empresas de call centers. A partir } \\
\text { disso, pode-se perceber a grande impor- } \\
\text { tância da discussão de temas que envolvam } \\
\text { esses trabalhadores, afinal estão sujeitos } \\
\text { constantemente a situações estressantes, } \\
\text { desgastes vocais, ruídos e posições inade- } \\
\text { quadas, as quais podem levar o trabalhador } \\
\text { à desenvolver doenças ocupacionais como } \\
\text { LER e DORT, além de ter uma influência } \\
\text { negativa na qualidade de vida. }\end{array}$ \\
\hline
\end{tabular}

A Ginástica Laboral (GL) vem se destacando pela fácil implantação, baixo custo e pelos resultados aparentemente positivos sobre a saúde de funcionários em atividade. Como afirma Mendes \& Leite (2004), a GL é um programa de qualidade de vida e de promoção do lazer, mesmo sendo realizada pelos trabalhadores durante o expediente de trabalho tem como objetivos iniciais melhorar a postura, promover o bem estar geral dos trabalhadores, diminuir o estresse ocupacional, além de tentar diminuir os acidentes de trabalho, aumentar a produtividade, prevenir as doenças ocupacionais e reduzir o número de afastamentos.

A GL quando aplicada através de monitores tem poder parcial de beneficiar os praticantes, pois o monitor não é instruído de forma completa ,ao contrário do acompanhamento de um profissional de Educação física ou profissional de fisioterapia que saberá como proceder, com capacitação adequada.

Candotti e Tarragô et al. (2012), consideram que a prática da GL proporciona aos trabalhadores a oportunidade de realizarem uma atividade física orientada por um profissional de Educação Física. Especula- se que esta aproximação possa gerar interesse, curiosidade e motivação nos trabalhadores para a prática regular da atividade física.

Segundo Martins \& Duarte (2000), além de orientar os exercícios laborais, o professor da GL também deve dedicar alguns minutos após as sessões para tirar dúvidas dos funcionários sobre atividade física e saúde, deixando-os mais seguros para realizar os exercícios em casa ou no trabalho e, até mesmo, difundir o conhecimento adquirido. Nesses poucos minutos o professor pode, inclusive, descobrir dificuldades imprevistas e reelaborar as sessões de GL para atender melhor o grupo, sendo este um ponto importante na dinâmica da GL para o sucesso do programa.

De acordo com os trabalhos analisados sobre GL em empresas de teleatendimento, os resultados de todos os estudos foram significamente positivos. Os trabalhadores tiveram um aumento do rendimento nas atividades diárias no trabalho, como também diminuição do estresse físico e mental, vícios posturais e lesões, e ao mesmo tempo em que a empresa estará investindo na saúde dos seus $\mathrm{k}$ trabalhadores, a mesma irá se beneficiar, com o baixo índice de faltas e atestados. 


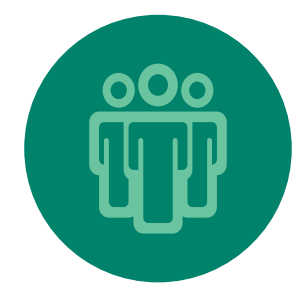

\section{CONCLUSÃO}

Por meio desta revisão pode-se sugerir que a Ginástica Laboral influencia na melhora das condições do bem-estar global dos indivíduos trabalhadores de empresas de teleatendimento, e que são necessários mais estudos na área da saúde do trabalhador de CallCenter, com uma elaboração metodológica mais completa, a fim de buscar novos conhecimentos para tornar a rotina do trabalhador mais saudável, pois trabalhadores sedentários que possuem uma jornada longa de trabalho, necessitam desta fonte de benefícios, para que ocorra a prevenção de possíveis lesões e afastamentos, por fim, as empresas devem adotar mais este meio, tendo assim uma diminuição em ambos problemas. 


\section{REFERÊNCIAS}

AZEVEDO, Pedro Galoza de, GRANADO, Shirley Orçai; A importância da ginástica laboral na prevenção de LER/DORT; a importância de la gimnasia laboral em la de las lesiones por EFDeportes.com, Revista Digital. Buenos Aires, Año 15, № 148, Septiembre de 2010.

BENEVIDES, LETÍCIA PEREIRA *, Análise da efetividade dos programas de ginastica laboral em empresas de teleatendimento, Universidade Federal de Londrina,2017.

CANDOTTI, Nome. Et al. Efeitos da ginástica laboral sobre a motivação para a Prática regular de atividade física. Revista Baiana de Saúde Pública, v. 35, n. 2, p.485, 2012.

CAÑETE I. Humanização: desafio da empresa moderna; a ginástica laboral como um caminho. Porto Alegre: Artes e Ofícios; 1996.

CARVALHO, Sérgio H. F. de. Ginástica laboral. Saúde em Movimento, 03 de janeiro de 2003.

International Society for Physical Activity and Health (ISPAH). The Toronto charter for physical activity: a global call to Action. Toronto: Global Advocacy Council for Physical Activity, International Society for Physical Activity and Health; 2010.

LIMA, Valquíria de ,Ginástica Laboral: Atividade Física no Ambiente de Trabalho. 1. Ed. São Paulo: v. 1. Phorte Editora, 2003. 240 p.

MARTINS CO, Duarte MFS. Efeitos da ginástica laboral em servidores da Reitoria da UFSC. Ver Bras Cien Mov. 2000;8(4):07-13.

MENDES AR, Leite N. Ginástica laboral: definições, classificações e objetivos. In: Mendes AR, Leite N. Ginástica laboral: princípios e aplicações práticas. São Paulo: Manole; 2004. p. 2-16.

MENDES, Ricardo Alves; LEITE, Neiva. Ginástica Laboral, Princípios E Aplicações Práticas. São Paulo: Manole, 2005.

OLIVEIRA, J. R. G.; SAMPAIO, Adelar Aparecido. A Ginástica Laboral na promoção da saúde e qualidade de vida do trabalhador. Caderno de Educação Física (UNIOESTE), v. 07, n. 13 p. 71-79, 2008.

RESENDE, et al*, Efeitos da ginástica laboral em funcionários de teleatendimento; ACTA FISIATR 2007; 14(1): $25-31$.

ROSSATO, Luana Callegaro;et al ;a ginástica laboral por trabalhadores das indústrias do Rio Grande do Sul, Brasil; RevBrasEducFís Esporte, (São Paulo) 2013 Jan-Mar;27(1):15-23.

SILVA AM. A regulamentação das condições de trabalho no setor de teleatendimento No Brasil: necessidades e desafios [tese]. Belo Horizonte: Universidade Federal de Minas Gerais; 2004.

TAUBERT, Fabiana Cristina de Freitas-Swerts; ROBAZZI, Maria Lúcia do Carmo Cruz; A GINÁSTICA LABORAL NA MINIMIZAÇÃO DO ESTRESSE OCUPACIONAL; Ver. Latino-Am. Enfermagem jul.-ago. 2014;22(4):629-36.

TSCHOEKE, Rony; COSTA, Lamartine Pereira da. Ginástica Laboral: Prerrogativa Do Profissional De Educação Física. São Paulo: Confef, 2015.

ZILLI, C. M. Manual de cinesioterapia/ginástica laboral: uma tarefa interdisciplinar com ação multiprofissional. São Paulo: Lovise, 2002. 